\title{
Le traitement et le recyclage de l'eau dans les industries agro-alimentaires
}

PAR

\author{
J. Sanquer \\ D.E.S.T., Ingénieur C.N.A.M., Chef du Secteur Centre \\ a l'Agence Financiere de Bassin Seine-Normandie
}

\section{L'axe d'intervention}

Depuis environ six années, les grandes options définies au Parlement lors de l'adoption de la loi sur l'eau du 16 décembre 1964, véritable cadre d'une politique rationnelle de préservation et de gestion de notre patrimoine hydraulique national, ont commencé à se matérialiser sur le terrain par des actions précises d'amélioration, en quantité et en qualité, de nos ressources en eau.

Dans cette vaste entreprise de lutte contre la pollution mise en place à l'échelon national, les six Agences Financières de Bassin ont inscrit, chacune avec un caractère particulier, leurs efforts dans un domaine spécifique, en fonction de l'urgence des problèmes à résoudre.

Pour le Bassin Seine-Normandie, au cœur duquel est implantée l'énorme métropole parisienne, les efforts de lutte contre la pollution des eaux souterraines et superficielles ont pris une ampleur toute particulière : l'alimentation en eau de toute l'agglomération parisienne se faisant à partir des eaux de surface, les grandes rivières de l'amont étant utilisées comme de véritables aqueducs.

Dans cette action pour maintenir les eaux superficielle: à un niveau de qualité nécessaire et suffisant pour pouvoir, après traitement, fabriquer une eau de consommation, il était et il demeure indispensable de s'attaquer aux principales sources de pollution implantées dans toute la partic amont du bassin.

Comme c'est dans cette zone géographique que se trouvent, d'une part les principaux établissements de l'industrie agro-alimentaire et que, d'autre part cette branche industrielle est particulièrement polluante (environ $30 \%$ de la pollution totale: MES + MO émise par les industries) l'action de l'Agence s'est spécialement exercée dans ce secteur de l'économie.

\section{Contraintes de situations et de marchés}

Dans ce secteur d'activité industrielle, l'action de l'Agence pour la réduction des flux polluants a dû revêtir un caractère particulier bien spécifique.

En effet, à part quelques unités qui ont été petit à petit rejointes par l'urbanisation d'une grande ville proche, comme dans la région de Reims ou de Châlons-sur-Marne, la quasi totalité des industries agro-alimentaires est, comme il se doit, implantée à la campagne.

De cette situation et de ce type d'activité essentiellement rurales, découle, sous l'angle de la pollution, toute une série de conséquences parmi lesquelles trois facteurs sont primordiaux :

- un rythme d'activité saisonnier (campagnes de 30 à 100 jours);

- un milieu récepteur hydraulique très souvent réduit (petite rivière voire un simple $\mathrm{ru}$ );

— une valeur ajoutée faible sur des prix définis à l'échelle européenne.

\section{Les atouts maîtres}

Essentiellement la place. De grandes surfaces disponibles auxquelles il convient d'ajouter, d'une part un flux polluant de nature oxydable non toxique, avec même parfois la possibilité de récupérer certains sous-produits à base de protéines qui peuvent sé révéler intéressants dans l'alimentation animale quand les conditions du marché le permettent.

De plus, ces dernières années, de vastes concentrations ont eu lieu dans ce secteur industriel, conduisant à une 
Extrait du tableau d'estimation forfaitaire

Sucrerie à partir de betteraves

Opérations de lavage, transport des betteraves et de diffusion :

a) Transport et lavage des betteraves sans décantation des eaux boueuses.

b) Transport et lavage des betteraves avec décantation efficace des eaux boueuses pendant plus de $24 \mathrm{~h}$.

c) Rejet des petites eaux en cas de diffusion discontinue.

Rejet des eaux d'écume après décantation pendant plus de $24 \mathrm{~h}$.

Rejet des effluents de régénération des résines du pro- $\mathrm{kg}$ de betteraves traitées. cédé Quentin.

Rejet des effluents de régénération des résines de démi- $\mathrm{kg}$ de betteraves traitées. néralisation des jus sucrés.

Rejet des eaux condensées d'évaporation des jus sucrés.

Raffinerie.

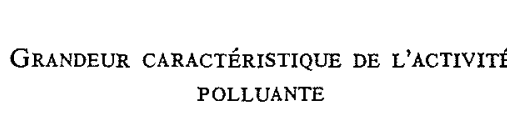

COEFFICIENT DE POLLUTION

$\mathrm{kg}$ de betteraves traitées.

$\mathrm{kg}$ de betteraves traitées.

0,9

1,6

$\mathrm{kg}$ de betteraves traitées, grandeur caractéristique accessoire.

\section{Production d'alcool}

Distillation industrielle de betteraves ou de mélasses pour la production d'alcool :

a) Transport et lavage des betteraves sans décantation litre d'alcool produit. des eaux boueuses.

$\mathrm{kg}$ de betteraves traitées.

$0,6 x^{(1)}$

1,6

\begin{tabular}{l|c} 
MES & MO \\
$(\mathrm{g})$ & $(\mathrm{g})$
\end{tabular}

b) Transport et lavage des betteraves avec décantation litre d'alcool produit. efficace des eaux boueuses pendant plus de $24 \mathrm{~h}$.

c) Rejet des petites eaux en cas de diffusion discontinue.

litre d'alcool produit, grandeur caractéristique accessoire.

Rejet des vinasses :

a) Rejet des vinasses brutes non concentrées sans litre de vinasses rejeté. récupération des levures.

b) Rejet des vinasses brutes non concentrées après litre de vinasses rejeté. récupération des saccharomyces.

c) Rejet des vinasses brutes non concentrées après litre de vinasses rejeté. récupération des torula.

(1) $x=$ Pourcentage des petites eaux non recyclées.

(2) $y=$ Pourcentage d'eaux condensées non réutilisées à des postes de fabrication où leur pollution est détruite.

Nora. - Pour ces activités où les installations de traitement peuvent être considérées comme faisant partie intégrante du procédé de fabrication, il est donné non seulement les coefficients spécifiques de pollution brute, avant ce traitement, mais aussi les coefficients spécifiques de pollution nette après ce traitement. 
augmentation importante des capacités de production de certains établissements; ces concentrations étant accompagnées, bien entendu, de la suppression de petites usines, si bien qu'actuellement, la taille moyenne d'une sucrerie, par exemple, se situe aux environs de $5000 \mathrm{t} / \mathrm{j}$.

Cette tendance facilite grandement les investissements antipollution. Etant donné la conjoncture mondiale dans ce domaine, il est vraisemblable que le phénomène ne fera que s'accentuer dans l'avenir.

A une échelle plus réduite une évolution analogue est en cours dans l'industrie de la levure ainsi que dans celle des produits amylacés.

\section{L'eau dans l'usine}

Dans ce type d'industrie, les usages de l'eau sont multiples. En effet, depuis l'arrivée au lavoir, par exemple des pommes de terre ou des betteraves, celle-ci va intervenir à peu près à tous les stades de la transformation :

- lavage des betteraves et tubercules;

- transport hydraulique de la matière première;

- procédés;

- refroidissement;

- transport des déchets.

Cependant, des calculs ont montré que l'utilisation de l'eau était directement fonction de l'abondance de celle-ci au niveau de l'établissement. En effet, en sucrerie par exemple, par tonne de betteraves entrant en fabrication, les valeurs oscillaient entre $1 \mathrm{~m}^{3}$ et $10 \mathrm{~m}^{3}$ selon le mode d'ali. mentation en eau de l'usine (chiffres de base 1968).

Depuis, la politique mise en place par les Agences a été déterminante sur les consommations unitaires, en particulier par une double incitation des redevances, tant au niveau du prélèvement qu'à celui de la pollution.

Bien évidemment, compte tenu des tarifs pratiqués, l'impact a été beaucoup plus fort dans les établissements alimentés uniquement à partir des nappes souterraines, l'exemple-type étant la Sucrerie de Cagny qui a atteint actuellement le chiffre record minimum d'environ 5001 par tonne de betteraves entrant en fabrication.

\section{La diversité des campagnes de fabrication}

Les années se suivent mais ne se ressemblent pas dans ces industries, essentiellement liées aux données climatiques; ces dernières vont influer tant sur la durée de la «campagne » que sur le flux polluant brut émis.

En effet, de fortes pluies ou du gel vont transformer complètement les conditions d'arrachage et conduire, au niveau du lavage, d'une part à des volumes d'eau beaucoup plus importants et surtout à des quantités de terre, «la tare » sans commune mesure d'une année sur l'autre.

C'est ainsi que certaines années, par exemple, il a été observé sur le lavoir, autant de terre que de betteraves ou de pommes de terre.

\section{L'origine de la pollution}

Qu'il soit externe ou de constitution, pour une partie en suspension (MES) et pour l'autre en solution (MO), le flux polluant émis est caractérisé par sa biodégradabilité.

- En sucrerie, les matières polluantes proviennent, lors du lavage et du transport des betteraves, des racines blessées, des radicelles, de l'humus (la partie de celui-ci collée à la betterave étant particulièrement riche en matières organiques).

Pendant le «process », le flux polluant provient des eaux de diffusion, des eaux de transport d'écume, des eaux de presses à pulpe..., avec en plus, pour certaines usines, des effluents de régénération de la déminéralisation des jus sucrés.

- En distillerie de betteraves, au niveau du lavage et du transport, les sources de pollution sont identiques à celles rencontrées en sucrerie.

Par contre, le rejet des vinasses épuisées est très polluant. Il peut atteindre $30000 \mathrm{mg} / \mathrm{l}$ de $\mathrm{DBO}_{5}$ pour 101 de vinasse en moyenne et par litre d'alcool produit.

Il est cependant possible de diminuer sensiblement le flux polluant émis en procédant à la récupération de certaines levures, en particulier les saccharomyces et les torula.

- En féculerie, les eaux de lavage et de transport des tubercules contiennent la quasi totalité des matières en suspension. Les eaux issues de l'extraction et du raffinage de la fécule, appelées "eaux de végétation », contiennent des matières solubles ou colloïdales, des sucres, des protéines, des acides gras, de la cellulose...

De caractère très fermentescible, les rejets polluants de cette industrie sont importants, étant donné les apports dus aux condensats des évaporateurs et aux eaux de lavage de l'amidon, soit environ $45 \mathrm{~g}$ de MES et $28 \mathrm{~g}$ de MO par kilo de produit entrant en fabrication.

- En levurerie, la production par fermentation de levures sélectionnées, sèches ou vivantes, à partir de mélasses provenant de sucreries, s'accompagne d'un rejet d'eaux résiduaires particulièrement chargées en matières oxydables, de l'ordre de $150 \mathrm{~g}$ par $\mathrm{kg}$ de mélasse traité.

\section{L'estimation forfaitaire du flux polluant}

Le tableau d'estimation forfaitaire du flux rejeté par les industries agricoles et alimentaires, a été bâti à l'aide de mesures effectuées par les différentes Agences concernées par ce type de problèmes, en particulier les Agences ArtoisPicardie et Seine-Normandie.

Ce barème, particulièremenı élaboré pour la sucrerie et la distillerie d'alcool de betterave, prend en compte des installations de traitement qui peuvent être considérées comme faisant partie intégrante du procédé de fabrication.

Il doit cependant être considéré comme représentant des flux polluants bruts minimum; en effet, par exemple il est 
évident que $81 \mathrm{~g}$ de matières en suspension par kilo de betteraves entrant en fabrication, est un chiffre particulièrement faible, seulement valable pour des années où l'arrachage a pu être effectué dans des conditions particulièrement favorables. D'autant plus que le mode d'extraction mécanique des betteraves conduit, d'une part à une augmentation très forte des matières en suspension et, d'autre part, à l'accroissement des matières oxydables dû aux chocs et brisures occasionnés par ce mode d'arrachage.

Dans l'avenir, il est vraisemblable que, progressivement, la mesure réelle du flux polluant viendra remplacer ce mode de calcul de l'assiette de la redevance, ne serait-ce que pour rendre homogène cette dernière avec le montant des aides financières que les Agences sont amenées à fournir pour éliminer les flux effectivement rejetés.

\section{QUELQUES EXEMPLES D'APPLICATION DES FORFAITS}

L'utilisation du barème ci-après conduit à une première estimation du flux polluant émis, avant traitement bien entendu; pour fixer les idées, voici deux exemples, l'un en sucrerie, l'autre en féculerie:

- Sucrerie de betteraves, pour l'activité transport et lavage.

Capacité de production : $5000 \mathrm{t} / \mathrm{j}$.

Transport et lavage des betteraves, sans décantation:

$$
\begin{array}{rrr}
\text { MES } & 81 \mathrm{~g} \times 5000000 \mathrm{~kg} / \mathrm{j} & =405000 \mathrm{~kg} / \mathrm{j} \\
\text { MO } & 2,9 \mathrm{~g} \times 5000000 \mathrm{~kg} / \mathrm{j} & =\frac{14500 \mathrm{~kg} / \mathrm{j}}{\text { Total. }}
\end{array}
$$

Après décantation efficace des eaux pendant $24 \mathrm{~h}$ :

$$
\begin{aligned}
& \text { MES } \quad 0,9 \mathrm{~g} \times 5000000 \mathrm{~kg} / \mathrm{j}=4500 \mathrm{~kg} / \mathrm{j} \\
& \text { MO } 1,6 \mathrm{~g} \times 5000000 \mathrm{~kg} / \mathrm{j}=8000 \mathrm{~kg} / \mathrm{j} \\
& \text { Total. } \quad \overline{12500 \mathrm{~kg} / \mathrm{j}}
\end{aligned}
$$

Dans l'exemple ci-dessus, la valeur du flux brut émis est très important, tout en étant vraisemblablement bien inférieure à la réalité. L'on voit aussi le rôle considérable que prend dans le forfait la décantation qui, à elle seule, fait chuter le flux de plus de $97 \%$.

- Féculerie de pommes de terre, pour l'activité de production de fécule de dextrine.

Matières premières entrant en fabrication : $1000 \mathrm{t} / \mathrm{j}$.

$$
\begin{aligned}
& \text { MES } \quad 45 \mathrm{~g} \times 1000000 \mathrm{~kg} / \mathrm{j}=45000 \mathrm{~kg} / \mathrm{j} \\
& \text { MO } \quad 20 \mathrm{~g} \times 1000000 \mathrm{~kg} / \mathrm{j}=20000 \mathrm{~kg} / \mathrm{j} \\
& \text { Total.. } \quad \overline{65000 \mathrm{~kg} / \mathrm{j}}
\end{aligned}
$$

Dans ce cas de figure, le flux brut résultant de la totalité des opérations de fabrication n'apparaît que dans une seule rubrique du barème; cependant, à partir de mesures spécifiques, il est apparu que la quasi totalité des matières en suspension était contenue dans les eaux de lavage des tubercules et que, par contre, les matières oxydables apparaissaient surtout dans les eaux dites de «végétation».

\section{Le point commun de ces activités industrielles : le recyclage}

Tant pour économiser la ressource au niveau du prélèvement que pour réduire le plus possible les volumes d'eaux résiduaires à traiter, il convient, bien entendu, en premier lieu, de réutiliser à «contre-courant » le maximum d'eau.

Pour cela, en particulier, tous les circuits de lavage ont été aménagés en utilisant des décanteurs-épaississeurs qui permettent, tout en diminuant la teneur des matières en suspension dans les circuits, d'obtenir, à la base des décanteurs, des boues dont la concentration peut atteindre 350 à $450 \mathrm{~g} / 1$.

Cette décantation peut être améliorée en utilisant, comme adjuvant, de la chaux et en équipant les circuits de désableurs et d'appareils de dégrillage.

Par ailleurs, tous les autres postes d'utilisation d'eau (depuis les eaux des condenseurs jusqu'à celles des pressesétoupes) ont été renvoyés en cascade vers le circuit de lavage.

Grâce à ces mesures systématiques, on a pu constater, en particulier sur les déclarations annuelles faites à l'Agence, que les prélèvements d'eau s'étaient réduits de façon notable.

C'est ainsi qu'un calcul récent a montré qu'actuellement la consommation moyenne d'eau ramenée à la tonne de betteraves, en sucrerie, s'établissait aux environs de $3,7 \mathrm{~m}^{3}$.

Cependant, la conséquence primordiale de cette politique au niveau de l'usine s'est surtout fait sentir sur le plan des volumes d'effluents rejetés.

Il est, en effet, capital de concentrer au maximum les eaux résiduaires pour :

- d'une part, réduire les capacités de stockage et,

- d'autre part, faciliter le traitement des effluents, ceux-ci demandant, par exemple, beaucoup moins d'énergie tant pour le transport que pour l'oxydation.

\section{Une nécessité : le stockage}

Pour réduire au maximum l'impact d'un rejet polluant massif produit pendant une courte période (celle d'une campagne oscillant entre 80 et 100 jours) débutant en septembre, à une époque de l'année où les rivières sont encore relativement proches de l'étiage, il importe de stocker les eaux résiduaires.

De nombreuses usines, dans le secteur des industries agro-alimentaires, pratiquent cette technique et stockent ainsi la totalité des effluents produits. Le rejet dans le milieu naturel peut alors être réalisé sur une très longue durée, pärfois sur les douze mois de l'année.

A condition bien entendu de disposer des capacités de stockage suffisantes, cette méthode permet aussi d'obtenir une amélioration naturelle de la qualité des eaux résiduaires.

Ce phénomène, qui se traduit par une forte diminution des matières oxydables, est essentiellement dû à l'autoépuration dans les bassins. Il a d'ailleurs été mis en évidence par de nombreuses études réalisées par l'I.R.I.S. (Institut de Recherches de l'Industrie Sucrière) et par celles qui ont été menées par l'Agence Artois-Picardie (Etudes de J. C. Noël, 1970/71). Voir figures 1,2 et 3. 


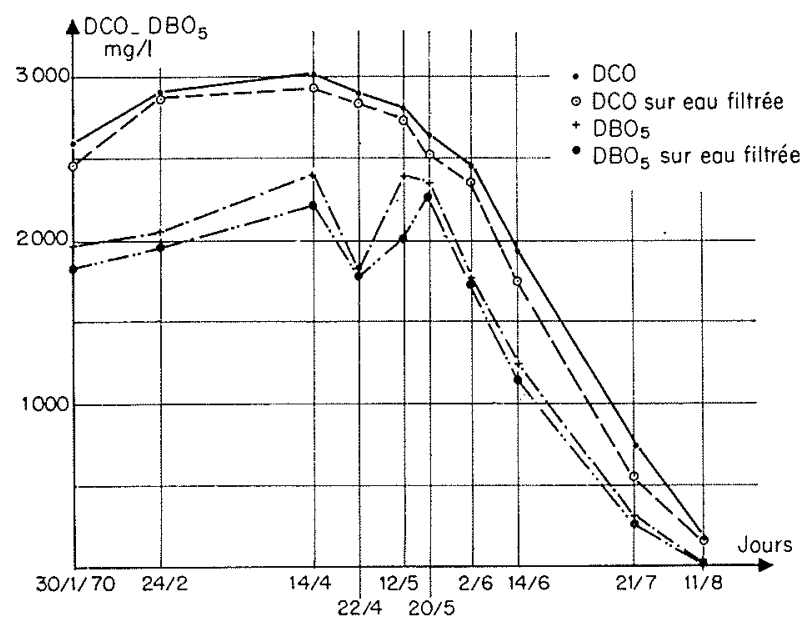

$1 /$ Sucrerie no 1.

Pour l'usine $n^{\prime 2} 1$, moyenne de $4,8 \mathrm{t}$ de $D C O$ par jour (minimum $2 \mathrm{t} / \mathrm{j}$, maximum $8,9 \mathrm{t} / j$ ) correspondant à des coefficients de biodégradation compris entre $0.01 \mathrm{~J}-1$ et $0,036 \mathrm{~J}-1$ (moyenne $0,023 \mathrm{~J}-1$ ). Si l'on rapporte ces valeurs au mètre carré de surface de bassin, on parvient à des valeurs théoriques de réaération comprises entre 15 et $67,02 \mathrm{~g} / \mathrm{j} / \mathrm{m}^{2}$ (moyenne : $0,36 \mathrm{~g} / \mathrm{j} / \mathrm{m}^{2}$ ).

Pour l'usine $n^{\circ} 2$, une moyenne de $3 \mathrm{t} / \mathrm{j}(0,4-5,1 \mathrm{t} / \mathrm{j})$ correspondant à des coefficients de biodégradation compris entre $0,002 \mathrm{~J}-1$ et $0,115 \mathrm{~J}-1$ (moyenne $0,0375 \mathrm{~J}-1$ ) ce qui donne des valeurs théoriques de la réaération rapportée au mètre carré de surface comprises entre 120 et $220 \mathrm{~g} / \mathrm{m}^{2} / \mathrm{j}$ (moyenne $164 \mathrm{~g} / \mathrm{m}^{2} / \mathrm{j}$ ) avec cependant deux valeurs faibles à 18 et $6 \mathrm{~g} / \mathrm{m}^{2} / \mathrm{j}$.

Pour l'usine $n^{n} 3$, une moyenne de $1,7 \mathrm{t} / \mathrm{j}(0,5-3,8 \mathrm{t} / \mathrm{j})$ correspondant à des coefficients de biodégradation compris entre 0,01 et $0,10 \mathrm{~J}-1$ (moyenne $0,042 \mathrm{~J}-1$ ) ce qui donne des valeurs théoriques de la réaération rapportée au mètre carré de surface comprises entre 14 et $106 \mathrm{~g} / \mathrm{J} / \mathrm{m}^{2}$ (moyenne $\left.46 \mathrm{~g} / \mathrm{j} / \mathrm{m}^{2}\right)$.

(Etude J. C. NOEL, Bulletin Seine-Normandie, n० 14, mars 1972).

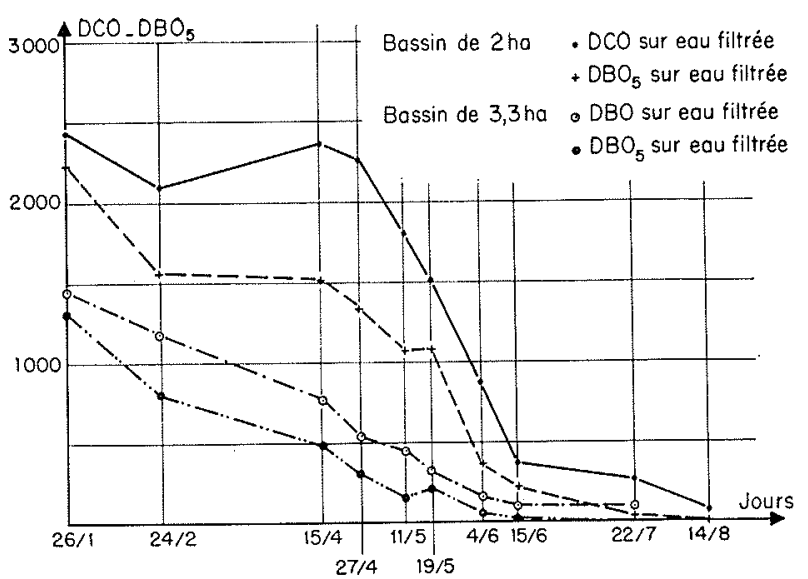

2/ Sucrerie no 2.

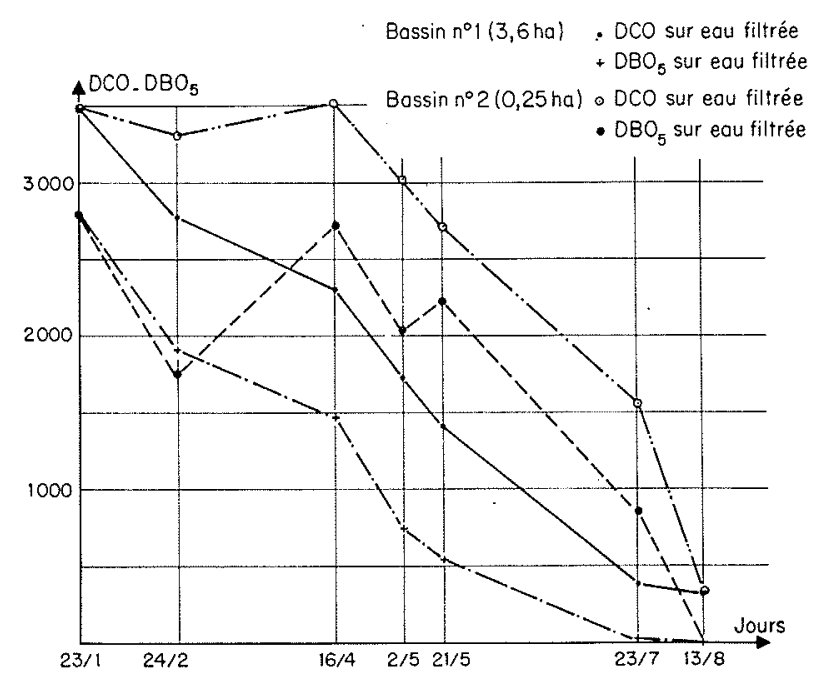

3/ Sucrerie no 3 .

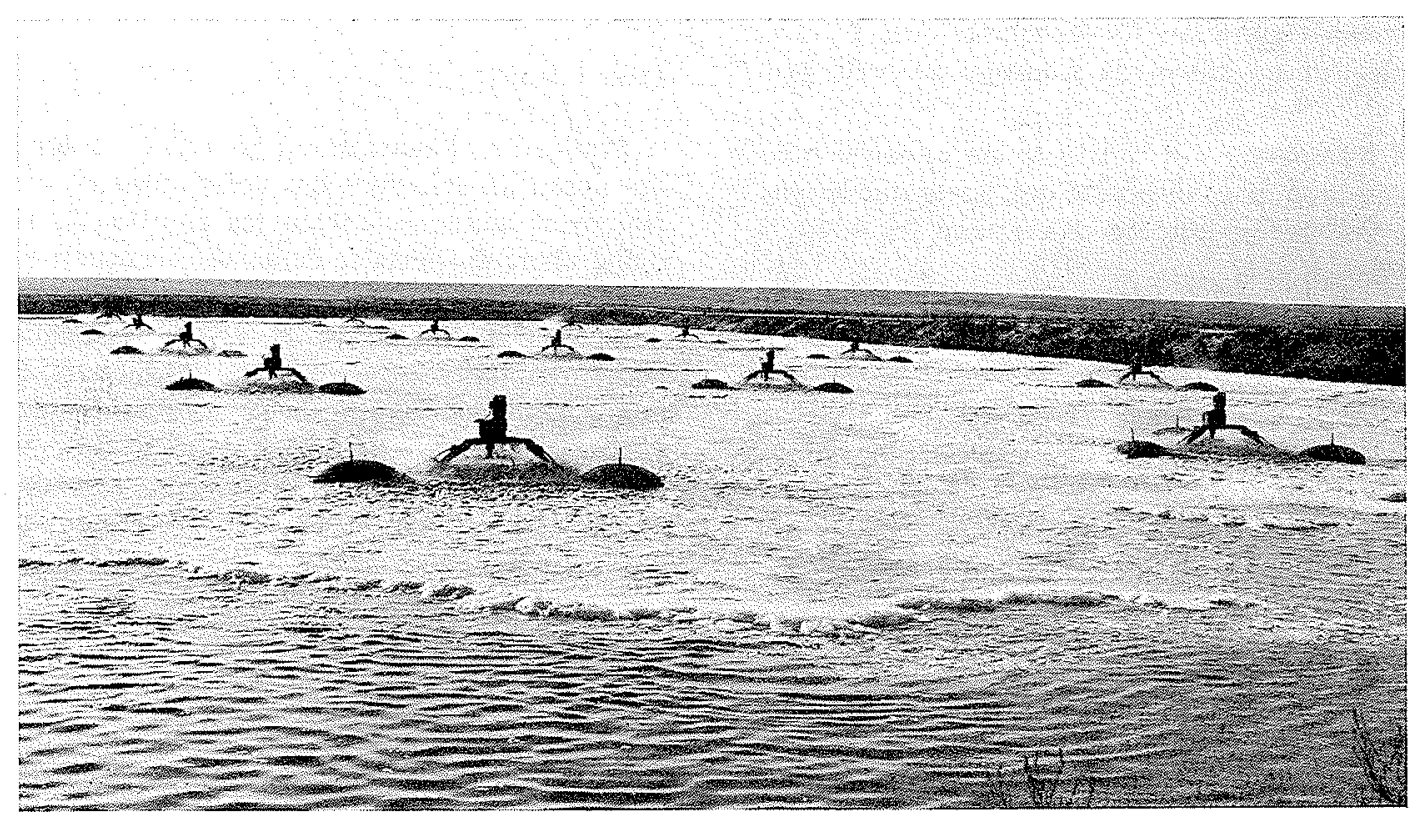

4/ Traitement d'eaux de sucrerie. Combinaison de lagunage naturel et de lagunage avec turbine. 1971 Sucrerie. Cooperative de Bazancourt (Marne). 
Dans la mesure, et cela est capital, où les bassins de stockage sont parfaitement étanches, mettant ainsi à l'abri les nappes aquifères sous-jacentes, il devient alors possible de procéder à un déstockage de l'effluent au fur et à mesure de l'amélioration de sa qualité.

C'est, par exemple, ce qui est pratiqué à la Sucrerie do Bresles (60) où toute la surface des bassins a été étanchée par un «beurrage » à l'argile et le rejet spécialement équipé d'un ouvrage hydraulique permettant de moduler tout au long de l'année, le fiux rejeté en fonction de la décroissance de la $\mathrm{DBO}_{\text {; }}$ dans les bassins.

\section{Les techniques de traitement}

\section{EN SUCRERIE}

Organisées autour et généralement en fonction des bassins de rétention, diverses méthodes d'épuration ont été mises en cuvre pour parvenir à des rejets polluants calculés selon la capacité du milieu récepteur dans lequel ils sont effectués.

C'est ainsi que l'on distingue, entre autres:

- le lagunage artificiel, par turbines de surface, qui vient en quelque sorte augmenter le processus naturel évoqué plus haut (voir fig. 4);

- l'utilisation de chenaux classiques d'épuration à brosses.

Dans le premier dispositif, le nombre, la puissance et le positionnement des turbines de surface sont déterminés, d'une part par les caractéristiques géométriques des bassins et, en particulier, par la profondeur de ceux-ci et, d'autre part, en fonction des objectifs de qualité à atteindre dans le milieu récepteur. Parfois même, la puissance des turbines a été prévue pour permettre une réutilisation de l'eau pour la campagne suivante.

Le second mode de traitement n'a, à notre connaissance, jusqu'ici été utilisé qu'à la Sucrerie de Sillery (51). Il consiste cn un petit chenal d'épuration fonctionnant toute l'année, en circuit fermé, sur un bassin de stockage de forte profondeur.

La nécessité d'employer des nutriants est apparue rapidement à l'exploitation de ce dispositif qui, après une longue mise au point, s'est révélé efficace et a atteint les $40 \mathrm{mg} / \mathrm{l}$ de $\mathrm{DBO}_{5}$ au rejet.

\section{EN FÉCULERIE}

Des techniques analogues, basées sur l'exploitation rationnelle des capacités de stockage, ont été mises en place. Cependant, dans cette industrie, les traitements biologiques aérobies extensifs doivent, à partir de $5000 \mathrm{mg} / \mathrm{l}$ de $\mathrm{DBO}_{\overline{5}}$, laisser la place à des techniques faisant intervenir un premier stade de digestion anaérobie contrôlée.

\section{EN LEVURERIE}

Jusqu'à présent, il nous semble que seule la concentration à multiples effets, a permis de réduire de façon importante les fiux polluants.

\section{L'épandage : une solution?}

On le rencontre sous toutes ses formes et cela dans tous les secteurs de l'industrie agro-alimentaire. Est-il la panacée ? Certainement pas, mais il permet, dans l'état actuel des techniques de l'épuration:

- soit d'éliminer des résidus difficiles à traiter, comme les vinasses de distillerie;

- soit de ramener sur les terrains les terres exportées lors de l'arrachage des betteraves ou des pommes de terre;

- soit de réaliser, dans quelques cas, de lirrigation.

Cette technique, associée ou non à un stockage de plus ou moins longue durée, est surtout pratiquée en SeineNormandie sous deux formes:

- l'aéroaspersion;

- et l'épandage par «billons».

\section{Par aéroaspersion}

Principalement en sucrerie, en féculerie et en distillerie, cette méthode, qui utilise un réseau "volant » de tuyauteries souples, disperse les eaux résiduaires à l'aide de "canons mobiles qui assurent, sur l'ensemble des terrains, une pluviométrie artificielle, judicieusement répartie en fonction de la capacité d'absorption des sols.

L'efficacité d'un tel dispositif, pour une surface donnée, est essentiellement dépendante, d'une part de la nature du sol et du sous-sol mais, d'autre part, plus encore des conditions climatiques et d'exploitation.

En effet, la pluviométrie naturelle, ou le gel par exemple, conditionne en grande partie le résultat de l'épuration. Il en va de même pour les rotations des canons qui doivent être constamment déplacés, de jour comme de nuit.

De ce fait, ce type de traitement entraîne des frais d'exploitation non négligeables, en particulier en maind'œuvre. Il réclame également de grandes surfaces.

\section{Par «billons》}

Pratiqué uniquement en sucrerie, ce mode d'épandage au niveau du sol, dans des voies appelées «billons», préalablement creusées suivant les courbes de niveau à l'aidc d'une charrue spéciale, demande beaucoup de préparation.

Par contre il assure, d'une part une excellente répartition de la terre sur les pièces de terrain et permet, d'autre part, en inter-campagnes au. moment des labours, de réaliser une bonne homogénéisation de la terre rapportée sur le sol en place.

Quel que soit le mode d'épandage, le résultat de celui-ci est directement lié :

- au bilan hydrique (eau rapportée + eau météorique -eau évapotranspirée);

- au bilan des apports et exportations minérales;

-- à la pédologie et

Jusqu'à présent, pour les Agences de Bassin, dans le calcul de la prime pour épuration, l'épandage est considéré comme exécuté correctement si les conditions suivantes sont réalisées :

- absence de ruissellement et de stagnation; 
- bon état d'exploitation agricole du terrain d'épandage;

- absence de mauvaise odeur.

A l'avenir, il est vraisemblable que ce domaine devra être étudié de plus près afin de déterminer, avec beaucoup plus de précision, l'impact de ces méthodes d'épuration sur le milieu naturel; des études dans ce sens sont en cours.

\section{La poursuite de l'action}

Après ce survol rapide, on peut constater que le bilan provisoire des efforts de l'Agence dans le domaine de l'industrie agro-alimentaire est loin d'être négligeable.

Elle a, en effet, participé financièrement à la réalisation de dispositifs d'épuration de tout type et parfois même à la récupération des sous-produits (protéines à la Féculerie de Vic-sur-Aisne).

Cette politique a déjà porté ses fruits, en particulier lors des étiages sévères de ces dernières années sur l'Oise et l'Aisne; les résultats ont pu être constatés par des mesures sur ces rivières.

En combinant son action à celle de l'Etat dans le cadre des contrats dits de «Branche » qui se développent actuellement dans le secteur des industries agro-alimentaires, elle a contribué grandement à l'élimination d'importantes sources de pollution.

$\mathrm{Si}$, jusqu'à présent, son action qui sera bien sûr poursuivie, a été de parer en quelque sorte au plus pressé, clans les années qui viennent la politique sera par contre infléchie vers la suppression des effets plus sournois de la pollution : en particulier, le dépistage des infiltrations, issues le plus souvent du manque d'étanchéité des dispositifs de stockage et d'épuration, sera entrepris.

\section{Discussion}

Président: M. D. MOYEN

M. Sanquer présente son exposé intitulé: Le traitcment et lo recyclage de l'eau dans les industries agro-alimentaires. M. le Président le remercie et propose de discuter cette communication en même temps que celle de M. Hrirot à qui il donne sans plus tarde: la parole; celui-ci présente son mémoire: La latte contre la pollution et le recyclage de l'eau dans la sidérurgie.

$$
*
$$

M. le Président remercie M. Sanquer et M. Hellot et ouvre la discussion sur leurs exposés respectifs.

M. Hellor demande à M. Sanquer si, dans linstallation de lavage des betteraves, la décantation el l'épuration des eaux boueuses sont réalisées dans le même bassin.

Généralement, répond M. SANQuer, on utilise un bassin divisé par une cloison en deux parties dinégales superficies; la décantation des matières en suspension dans l'eau est réalisée dans le «grand bassin» lequel alimente par déversement le «petit bassin» où se fajt l'épuration.

M. MarÉcaux (Sté Spéchim) souhaiterait obtenir quelques renseignements sur l'épandage par aspersion des eaux résiduaires de sucreries ou de distilleries. Quelle est la superficie nécessaire pour traiter un volume (ou un débit) d'eau déterminé ?

Ces superficies sont très importantes, répond M. Sanquer. Ainsi la féculerie Doitteau à Haussimont, qui transforme actuellement 1200 à 1300 tonnes de pommes de terre par jour réalise l'épandage sur environ 300 hectares; mais, afin d'assurer une «rotation s sur trois ans, les champs d'épandage s'étendent sur un ensemble de 900 hectares dont 300 sont utilisés environ 100 jours all cours d'unte année, le solde, soit 600 hectares restant en jachère. En comptant sur un débil deau résiduaire de 2 à $3 \mathrm{~m}^{3}$ par tonne de pommes de terre entrant en fabrication, on arrive à un débit d'épandage de 2000 à $3000 \mathrm{~m}^{3} /$ jour pendant 100 jours (soit au total 2 à $300000 \mathrm{~m}^{\text {* }}$ par an).

Quelle est linfluence du climat et de la teneur en matière: oxydables des eaux d'épandage sur le rythme et la qualité de l'opération? Comment surveiller l'infiltration pour éviter la pollution des nappes souterraines? demande M. MaréchuX.

En pratique, répond $M$. Helror, on fixe les débits moyens a épandie par hectare. Ils résultent de l'étude du climat (surtout de la pluviométrie) de la région, de la perméabilité du sol, des façon; culturales, etc.
Un autre facteur limitant de ce type d'épandage, précise M. DanIII., est la quantité de matières organiques qui peut être assimilée par les organismes vivants et les plantes pendant une période donnée. De toute façon, malgré l'évapotranspiration, il subsiste généralement un risque de voir une partie des eaux d'épandage, insuffisamment épurées, atteinure les nappes souterraines. Aussi les autorisations d'épandage font-elles l'objet d'une réglementation et d'une surveillance spéciales. En conclusion, les techniques d'épandage restent aléatoires, mal maitrisées et peuvent être dangereuses dans certains cas.

11 existe, observe $M$. le Président, des périmètres de protection autour des points de prélèvements d'eau souterraine destinée à lalimentation humaine (voir, notamment larticle 7 de la loi dil 16 décembre 1964) et des échantillons d'eau sont prélevés régulicrement pour contrôler ics qualités des eaux captées.

M. MarÉcaux craint que la propagation des odeurs provenant des champs d'épandage conduise à des nuisances sérieuses pour l'environnement.

Les vinasses de distillerie peuvent poser quelques problèmes à cet égard, dit M. Sanquer, mais, jusqu’à présent, les terrains d'épandage sont loin des agglomérations et même des habitations.

De toute façon, conclut $M$. le Président, les droits des tiers demeurent réservés et en cas de conflit les tribunaux sont seuls juges.

M. le Professeur Carta (Cagliari) demande s'il se forme des écumes dans les bassins de décantation et d'épuration; dans l'affirmative, comment s'en débarrassetton?

Suivant les vents dominants, répond $M$. Sanquer, les écumes ont tendance à se concentrer dans certaines régions des bassins; dans l'une des sucreries contrôlées, elles sont reprises par une petite pompe et mélangées aux eaux d'épandage.

Sur une question de M. le Professeur CARTA, M. Hellot précise que les eaux huileuses subissent un traitement spécial qui comporte - après mesure du pH - la séparation de la phase huileuse par flottation ou électroflottation. Les huiles ainsi récupérées sont centrifugécs puis incinérées car elles ne sont pas réutilisables économiquement.

M. Françors suntéresse à l'étanchéité des bassins de décantation ou de "lagunage » creusés dans le sol; les sédiments déposés n'ont-ils pas tendance à renforcer l'étanchéité initiale d'un bassin ne comportant pas de revêtement.

D'un échange de vue sur ce point entre M. Sanquer, M. Hellot et M. RéméNIÉRAs, il ressort que chaque bassin a un comportement 
particulier qui dépend de la perméabilité du terrain, de la composition des dépôts et de la position de son plan d'eaul par rapport à la nappe phréatique. $\mathrm{Si}$, à certaines saisons, la nappe alimente le bassin, il peut en résulter une destruction plus ou moins complète di colmatage du terrain (et des dépôts) qui a pu se réaliser peu à peu au cours des périodes où leau du bassin s'infiltrait vers la nappe.

Dans la seule sucrerie de l'Est où un bassin de ce genre ne comportait pas d'exutoire, signale M. Hellot, on a observé que le niveau du plan d'eau dudit bassin baissait d'une campagne à l'autre; l'évaporation naturelle étant faible par rapport aux précipitations de la période considérée, on en déduit qu'il y a des infiltratioiss vers la nappe (ce qui n'est peut-être pas sans danger).

Les procédés biologiques sont-ils les seuls utilisés pratiquement pour la dégradation des phénols contenus dans les eaux résiduaires? demande M. SERPAUd.

Industriellement, répond $M$. Hellor, je ne pense pas qu'il existe à l'heure actuelle d'autre technique; mais en laboratoire, on a étudiś différentes possibilités, en particulier l'utilisation de l'ozone, du chlore, etc.; pratiquement, une investigation complète de ces procédés a été faite mais il n'en existe pas d'application dans l'industrie en raison de leurs cô̂ts non compétitifs.

A-t-on fait des recherches sur la flottation ionique, interroge M. le Professeur CarTa?

Des essais utilisant des résines échangeuses d'ions ont été réalisés, répond M. Hellot, mais celles-ci sont très rapidement « empoisonnées », ce qui semble condamner ce procédé pour des raisons économiques.

En ce qui concerne les eaux ammoniacales des cokeries, nous souhaitons voir mettre au point, dans l'avenir, une méthode permettant d'éliminer tous les polluants. Par exemple, une méthode telle que celle faisant appei à la filière: distillation, entraînement à la vapeur, concentration et, après concentration, soit incinération, soit récupération des polluants (si cette dernière s'avère rentable).

Le Président clôt la discussion sur ces deux premiers mémoires et donne la parole à $\mathrm{M}$. Daniel pour l'exposé de sa communication.

\section{Abstract \\ Water treatment and recycling in the agricultural and food industries}

The contents of this paper relate to pollution control as envisaged in a law on water management adopted by French Parliament w.e.f. 16.12.64, implementation of which has taken the form of definite forms of action with a view to both quantitative and qualitative improvement of available water supplies. It is submitted with the following objects in view:-To examine the amount of pollution caused by effluent from agricultural and food factories in the Seine-Normandy region above Paris;

-To review the purification methods most frequently applied.

The limitations, requirements and "trump cards" associated with factory positions in the considered region are described (especialiy in relation to local natural outlets), also how this policy stands with regard to the European market.

Emphasis is laid upon the original features and limitations of the proposed pollution control methods, especially the following:-
- Recycling of water to reduce effuent discharge;

-Storage facilities;

-Natural self-cleaning action during storage (re-aeration).

-Recent artificial re-aeration methods;

-Effluent-spreading methods.

The basic role of natural self-purification is considered from the two following angles:-

-Reduction of effluent volume by recycling;

-Rational storage capacity management based on "modulation" of effluent discharge in keeping with water quality improvement during the year.

Author concludes with a discussion of French Government contract clauses shortly to be agreed upon with the various sectors of the agricultural and food industry concerned, which lay down the main guidelines for pollution control policies to be applied in years to come. 